\title{
The integration and management of noncommunicable diseases in primary health care
}

Slim Slama ${ }^{1}$, Asmus Hammerich ${ }^{1}$, Ahmed Mandil ${ }^{2}$, Abla Mehio Sibai ${ }^{3}$, Jaakko Tuomilehto ${ }^{4}$, Kremlin Wickramasinghe ${ }^{5}$ and Tom McGee ${ }^{1}$

${ }^{1}$ Division of Noncommunicable Diseases and Mental Health, WHO Regional Office for the Eastern Mediterranean, Cairo, Egypt. ${ }^{2}$ Division of Information, Evidence and Research, WHO Regional Office for the Eastern Mediterranean, Cairo, Egypt. ${ }^{3}$ Department of Epidemiology and Population Health, American University of Beirut, Beirut, Lebanon. ${ }^{4}$ Research Division, Dasman Diabetes Institute, Dasman, Kuwait. ${ }^{5}$ Centre on Population Approaches for NCD Prevention, Nuffield Department of Population Health, University of Oxford, Oxford, United Kingdom.

Citation: Slama S, Hammerich A, Mandil A, Sibai AM, Tuomilehto J, Wickramasinghe K, et al. The integration and management of noncommunicable diseases in primary health care. East Mediterr Health J. 2018;24(1):5-6. https://doi.org/10.26719/2018.1.5

Copyright (C) World Health Organization (WHO) 2018. Some rights reserved. This work is available under the CC BY-NC-SA 3.0 IGO license (https:// creativecommons.org/licenses/by-nc-sa/3.0/igo)

Noncommunicable diseases (NCDs) are a great burden in the Eastern Mediterranean Region (EMR) and, if no strategic intervention is taken, the burden is forecast to become even heavier, particularly with the additional impact of ageing populations. Currently, $62 \%$ of deaths in the EMR are due to NCDs (1). However, by 2030 this proportion is projected to increase to nearly $70 \%$ (2). The EMR is disproportionately affected by NCDs as a result of the Region's rapid urbanization and the globalization of unhealthy behaviours (3). Moreover, many of the EMR countries have health systems whose structures are not designed for the prevention, early detection and management of NCDs.

Indeed, the World Health Organization's (WHO) 2016 'Framework on integrated, people-centred health services' (4) emphasized the importance of organizing primary health care $(\mathrm{PHC})$ around the comprehensive needs of people, rather than around a singular focus of specific diseases. When combined with population-wide preventative measures, people-centred PHC can prove very effective in tackling NCDs both at the population and individual level. The chronic nature and multiple comorbidities in people with NCDs are targeted by the core principles of people-centred $\mathrm{PHC}$ (5). The family practice model of people-centred care must be continuous, that is, the patient/client returns to the same health worker over time; the care should be accountable, with each health team responsible for a defined population; close-toclient care ensures improved access to $\mathrm{PHC}$ within the community; and care must be coordinated, with the PHC acting as the first point of entry and referring patients/ clients to other levels of the health system where necessary. Such a model promotes improved prevention as well as the early detection and management of NCDs. It enables health workers to work closely with people with a high risk of developing NCDs and treat those with the early stages of a disease, thereby preventing the disease from advancing or developing complications. This is also an ideal environment for the implementation of cost-effective brief interventions such as WHO recommended 'Best Buys' (6). If required, PHC workers can then efficiently refer on patients/clients who require specialist care.
Thus, PHC represents the most appropriate and equitable framework for implementing individual health care interventions for NCDs (7). On a regional level, WHO Regional Office for the Eastern Mediterranean (EMRO) has also acknowledged the need for family practice to address NCDs and, beyond that, advance universal health coverage (UHC) $(8,9)$. However, challenges to the integration of NCDs in PHC remain, not least due to the diversity among and within the EMR's 22 countries, due to various socio-economic and logistic reasons.

Despite the differences in health system performance and level of health expenditure, the service delivery of NCD-orientated PHC is a constraint common to each of the EMR's Member States. The Region's PHC systems have traditionally focused on communicable diseases, other acute conditions and maternal and child health. Primary health care was consequently arranged vertically and organized according to specific services. Patients/clients might be attending a different health professional each time they visit the clinic. This structure was incompatible with the need to develop a holistic appreciation of the PHC visitor, his or her health conditions and lifestyle throughout the life course. Therefore, a transition has begun away from the vertical, disease-specific approaches of the past and towards broader people-centred care.

Any effort to integrate NCDs in PHC must firstly identify the key interventions and cost-effective services that can be implemented. This led to the 2010 launch of the 'WHO Package of Essential Noncommunicable Disease Interventions for Primary Health Care' (WHO PEN) (10). The WHO PEN is composed of a prioritized set of measures that address the four major NCDs (cardiovascular diseases, cancer, diabetes, and chronic respiratory disorders) and their risk factors. Secondly, a means of ascertaining the capability of each country's PHC system and ways of improving this system are also required. In view of this, EMRO has developed the 'Framework on Strengthening the Integration and Management of Noncommunicable Diseases in Primary Health Care' (11). Adopting a health systems perspective, the Framework provides EMR countries with key action areas, tailored to NCDs, for each of the six building blocks (governance; financing; health workforce development; 
service delivery; essential medicines and technologies; and health information) (12). Some recommended interventions, such as the need to support community-based training and self-care, are applicable to all countries. Other interventions target specific countries, depending on the challenges they face. For instance, countries in the midst of conflict require the development of a resilient PHC system. More developed countries may need to focus on health information systems, including electronic health records to link individuals' data across PHC and higher levels of care.

Early progress in integrating NCDs in PHC has focused on cardiovascular diseases (CVD). This is in large part because two of the WHO 'best buys' (6) and two of the nine global NCD voluntary targets (13) relate to CVD. As well as offering recommended interventions, the EMR Framework also allows a situational analysis of each country to be carried out, as in a 2016 review of the nationally adapted PEN initiative in the Islamic Republic of Iran (IraPEN). The Framework-based analysis identified short-, medium- and long-term health system reforms for the optimal integration of NCDs into PHC. When considering the challenges that lie ahead, it should be remembered that NCD integration in people-centered PHC cannot occur in isolation; instead it should be part of wider health sector reform that is a continuous process. This is the case for several EMR countries currently expanding their essential service delivery package, models of care and healthcare financing. Thus, integrated people-centred PHC services form a crucial component of strong health systems and are vital for moving closer to UHC, especially in the Eastern Mediterranean Region.

\section{References}

1. World Health Organization. Global health estimates 2015: Deaths by cause, age, sex, by country and by region, 2015-2000. Geneva: World Health Organization; 2016. (http://www.who.int/healthinfo/global_burden_disease/estimates/en/indexı.html).

2. World Health Organization. Projections of mortality and causes of death, 2015 and 2030. Geneva: World Health Organization; 2013 (http://www.who.int/healthinfo/global_burden_disease/projections/en/).

3. World Health Organization, World Heart Federation, World Stroke Organization. Global atlas on cardiovascular disease prevention and control. Geneva: World Health Organization; 2011 (http://www.who.int/cardiovascular_diseases/publications/ atlas_cvd/en/).

4. World Health Organization. Framework on integrated, people-centred health services (A39/69). Geneva: World Health Organization; 2016 (http://apps.who.int/gb/ebwha/pdf_files/WHA69/A-39_69en.pdf?ua=1).

5. World Health Organization. The world health report. Geneva: World Health Organization; 2008 (http://www.who.int/whr/2008/ whro8_en.pdf).

6. World Health Organization. Tackling NCDs. 'Best buys' and other recommended interventions for the prevention and control of noncommunicable diseases. Geneva: World Health Organization; 2017 (http://apps.who.int/iris/bitstream/1/259232/10665/WHONMH-NVI-17.9-eng.pdf?ua=1).

7. World Health Organization. Global status report on noncommunicable diseases 2010. Geneva: World Health Organization; 2011 (http://apps.who.int/iris/bitstream/9789240686458/1/44579/10665_eng.pdf).

8. World Health Organization. Framework for action on advancing universal health coverage (UHC) in the Eastern Mediterranean Region. Geneva: World Health Organization; 2016. (http://applications.emro.who.int/docs/Technical_Notes_EN_16287.pdf?ua=1).

9. WHO Regional Office for the Eastern Mediterranean (EMRO). Conceptual and strategic approach to family practice. Cairo: EMRO; 2014_http://applications.emro.who.int/dsaf/EMROPUB_2014_EN_1783.pdf).

10. WHO Regional Office for the Eastern Mediterranean (EMRO). Package of essential noncommunicable (PEN) disease interventions for primary health care in low-resource settings. Cairo: EMRO; 2010 (http://www.who.int/nmh/publications/ essential_ncd_interventions_lr_settings.pdf).

11. WHO Regional Office for the Eastern Mediterranean (EMRO). Report on the regional meeting on strengthening the integration and management of noncommunicable diseases in primary health care. Cairo: EMRO; 2014 (http://applications.emro.who.int/ docs/IC_Meet_Rep_2014_EN_15646.pdf).

12. World Health Organization. Monitoring the building blocks of health systems: a handbook of indicators and their measurement strategies. Geneva: World Health Organization; 2010 (http://www.who.int/workforcealliance/knowledge/toolkit/26.pdf?ua=1).

13. World Health Organization. NCD global monitoring framework. Geenva: World Health Organization; 2011 (http://www.who.int/ nmh/global_monitoring_framework/en/). 